\title{
Hematocrit level correlates with lungs resistivity in elderly patients with cardiogenic pulmonary edema
}

\author{
Marina Arad', Avraham Adunsky ${ }^{1}$, Sharon Zlochiver², Ofer Barnea ${ }^{2}$, Shimon Abboud ${ }^{2}$ \\ ${ }^{1}$ Department of Geriatric Rehabilitation Sheba Medical Center, Tel-Hashomer, and Sackler Faculty of Medicine, Tel Aviv University, \\ Tel Aviv, Israel; ${ }^{2}$ Department of Biomedical Engineering; Tel-Aviv University, Tel Aviv, Israel. \\ Email: aradm@post.tau.ac.il
}

Received 17 September 2010; revise 22 September 2010; accepted 26 September 2010.

\begin{abstract}
Regular monitoring of pulmonary congestion in cardiogenic pulmonary edema (CPE) patients is necessary for its adequate management via pharmaceutical treatment. It is well known that the development of CPE is accompanied with an increase in hematocrit, plasma protein concentration and colloid osmotic pressure due to the decrease in the plasma volume. In the present study the mean left and right lung resistivity values taken pre- and post treatment with diuretics using a hybrid bio-impedance electrical impedance tomography system were correlate to the measured changes in hematocrit level. A marginal significant correlation was found between the absolute mean lung resistivity and hematocrit levels (Pearson's correlation coefficient of $R=0.4$, $p$-value $=$ $0.057)$. When the change in the mean lung resistivity of a patient was plotted vs. the change in hematocrit readout, a significant linear correlation was found $(R=$ 0.7 , p-value $=0.02$. These results support the validity of the resistivity measurements using bio-impedance system in monitoring changes of pulmonary edema in CPE patients.
\end{abstract}

Keywords: Bio-impedance; Parametric EIT; Cardiogenic Pulmonary Edema; Hematocrit

\section{INTRODUCTION}

Congestive heart failure and a consequent CPE are a major health problem in the western world. Cardiogenic pulmonary edema (CPE) is a pathological condition in which extravasation of fluid and colloid from the pulmonary capillaries into the interstitium and alveoli of the lungs occurs as a result of increased hydrostatic pressure in these capillaries that exceeds the plasma oncotic pressure. This happens when the heart's function is impaired (heart failure) in conditions such as pulmonary venous outflow obstruction, left ventricular failure, ischemic heart disease, myocardial infarction or left atrial myxoma tumor, to the effect that blood cannot be sufficiently pumped in proportion to the tissues' metabolic demand. As a result, a compensatory increase in pulmonary venous pressure is developed $[1,2]$.

The development of CPE is accompanied with an increase in hematocrit, plasma protein concentration and colloid osmotic pressure due to the decrease in the plasma volume. In a study conducted on $95 \mathrm{CPE}$ patients and 71 control subjects, a significant difference in hematocrit level was found between the two groups (44.3 vs. $42.1 \%$ for the CPE and control groups, respectively). Moreover, a gradual decrease in the hematocrit level was observed for the CPE group during therapy for those patients whose condition was improved post-treatment (from $44.5 \pm 0.8$ to $41.3 \pm 0.8 \%, \mathrm{n}=65$ ) [3]. Similar results were observed by the same research group in an additional study [4]. In that study, the effect of CPE therapy using furosemide, morphine and oxygen on various plasma parameters was measured in $\mathrm{CPE}$ patients. A statistically significant decrease in hematocrit from $42.8 \pm 1.9$ to $36.7 \pm 1.8 \%$ was found after an average of 21.3 hours of treatment $(n=10$, $p$-value $<0.001)$. This decrease was in conjunction with a difference between the urine output and fluid intake of $2.823 \pm 0.8481$. Further demonstration for the correlation between the lungs fluid volume and hematocrit was given in another study conducted on a group of $10 \mathrm{CPE}$ patients that were treated with large doses on intravenous furosemide [5]. In that study, hemodilution was detected 2 hours after the start of treatment, resulting in a significant reduction of blood viscosity and hematocrit. The hematocrit was reduced by a mean of $3 \%$ and $5.4 \%$ at 30 and $120 \mathrm{~min}$ utes after the start of treatment.

The gradual filling of the lungs with fluids during the development of CPE results in substantial changes in the electric impedance of the lungs, as the lung fluids exhibit higher conductivity in relation to the electrically insulating air. Since the lungs are the largest in volume organ in 
the thoracic volume, such impedance changes are expected to be large enough to be non-invasively detected on the body surface via impedance measurement. Therefore, the bio-impedance technique may be utilized as an alternative non-invasive CPE monitoring method.

We previously reported on a newly developed hybrid EIT system (CardioInspect, Tel-Aviv University, Israel) that combines principles from the bio-impedance and EIT techniques and enables the reconstruction of the separate left and right lung resistivity values. The system's performance was studied on both healthy and CHF patients, and its capability to diagnose and monitor patients was demonstrated [6-10]. Here we examine the correlation between the measured left and right lung resistivity values to the hematocrit level in a group of CHF patients during diuretics treatment.

\section{METHODS}

\subsection{Clinical Study}

A clinical study was conducted in the geriatric department in Tel-Hashomer hospital, Ramat-Gan, Israel. The study included $12 \mathrm{CHF}$ patients $(\mathrm{n}=4 / 8$, male/female, mean age $78 \pm 10$ years) that were diagnosed and found having pulmonary edema and were under monitoring and CPE management. All participants signed an informed consent form, and the study was approved by the local Helsinki committee. Two bio-impedance measurements were taken. The first measurement was taken as a reference measurement to determine the lungs' resistivity value pre-treatment. The second measurement was taken following treatment with diuretics, morphine and oxygen. In addition to the bio-impedance measurements, blood samples were taken at the two measuring times, and hematocrit level was assessed as part of a complete blood count. All bio-impedance measurements were taken shortly after the patients were asked to sit rested to ensure shallow tidal respiration and minimal body movements that may impair reproducibility of the results.

\subsection{Bio-impedance System}

We employed the portable PulmoTrace hybrid bio-impedance measurement system (CardioInspect, Tel-Aviv University, Tel-Aviv Israel). The system has been previously studied and described in detail [6-10]. Briefly, the system comprises of three units (Figure 1): 1) an 8 -electrode belt is attached around the patient's chest at the plane of the fifth intercostals space in the midclavicular line. The electrodes are $\mathrm{Ag} / \mathrm{AgCl}$ disposable, and they are attached at equal angular distance from each other using 8 adjustable elongation segments on the belt; 2) an analog unit generates the injection current ( $3 \mathrm{~mA} \mathrm{ptp}, 20 \mathrm{kHz}$ ) and directs the current through a

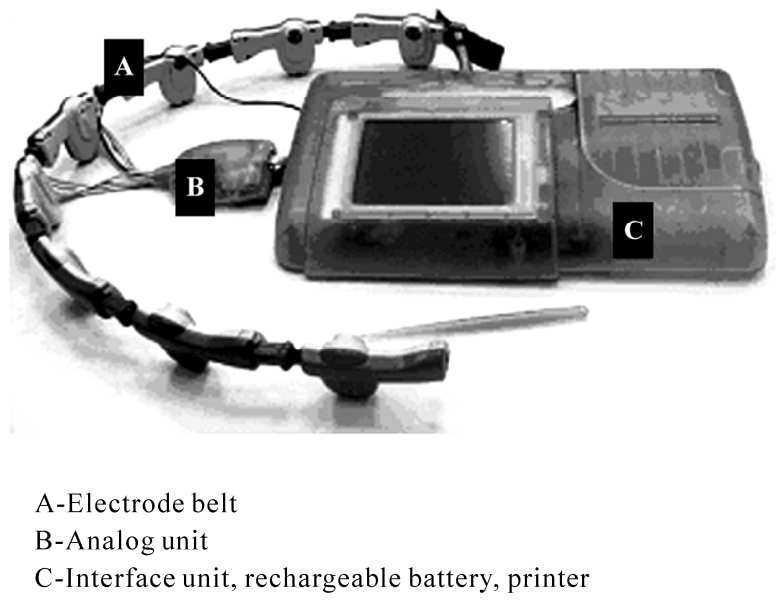

Figure 1. PulmoTrace hybrid bio-impedance measurement system.

demultiplexer to the appropriate pair of electrodes. The developing voltages at the rest of the electrodes are measured differentially, amplified, filtered and digitized for analysis. The current injection is done in the opposite injection scheme, i.e. a total of 4 injections is performed, for each 5 voltages are measured with the remaining 6 electrodes, yielding 20 independent measurements; and 3 ) an interface unit includes a microprocessor that processes the digitized voltage measurements and solves a parametric inverse-problem to estimate the left and right lung resistivity values. The reconstruction algorithm is based on an iterative, parameterized Newton-Raphson inverse solver as previously reported [6]. The results are shown on an LCD screen, and can be printed out. The system is powered by a rechargeable battery to ensure electrical safety in the hospital environment. A custom ECG signal is taken by the system prior to the bio-impedance measurements, in order to synchronize the current injections and surface voltage measurements to the iso-potential phase of the ECG, so that to ensure a similar heart geometrical configuration between measurements. The entire measurement procedure lasts less than 1 minute and completely painless.

\subsection{Statistics}

Data were fitted using linear regression, and Pearson's correlation coefficient was computed to check the strength of linear dependence. The significance of correlation was assessed using a transformation into t-statistics. A p-value of less than 0.05 was considered statistically significant.

\section{RESULTS}

Table 1 summarizes the bio-impedance and hematocrit measurements that were taken for all 12 patients at the two measuring phases-pre-treatment (reference) and 
Table 1. Bio-medical and hematocrit level measurements.

\begin{tabular}{ccccccc}
\hline Patient \# & Sex & Age & \multicolumn{2}{c}{ Reference measurement } & \multicolumn{2}{c}{ Post-treatment measurement } \\
\hline & & & $\begin{array}{c}\text { Mean lung resistivity } \\
{[\Omega \text { cm }]}\end{array}$ & $\begin{array}{c}\text { HCT Level } \\
(\%)\end{array}$ & $\begin{array}{c}\text { Mean lung resistivity } \\
{[\Omega \text { cm }]}\end{array}$ & $\begin{array}{c}\text { HCT Level } \\
(\%)\end{array}$ \\
1 & F & 92 & 853 & 38.6 & 800 & 39.6 \\
2 & M & 80 & 1083 & 44.9 & 1231 & 43.1 \\
3 & F & 91 & 824 & 31 & 889 & 33.2 \\
4 & F & 73 & 715 & 33.3 & 834 & 36.9 \\
5 & M & 69 & 625 & 38.2 & 675 & 36.1 \\
6 & F & 69 & 861 & 29.2 & 1200 & 38 \\
7 & F & 88 & 1060 & 35.8 & 1061 & 33.2 \\
8 & F & 77 & 771 & 34.5 & 852 & 33.6 \\
9 & F & 83 & 660 & 28.3 & 753 & 30.5 \\
10 & M & 84 & 884 & 41.5 & 935 & 41.5 \\
11 & F & 81 & 996 & 35.1 & 1089 & 38.1 \\
12 & M & 58 & 871 & 43.4 & 859 & 44.3 \\
\hline
\end{tabular}

post-treatment. A two-sample paired t-test showed that the mean lung resistivity was significantly different between the two measurements: $850.3 \pm 145.6$ vs. $931.5 \pm$ $176.1 \Omega \mathrm{cm}$, pre- and post-treatment, respectively ( $\mathrm{p}$-value $=0.016)$; on the other hand, the hematocrit levels between the measurements were not significantly different: $36.2 \pm 5.4 \%$ vs. $37.3 \pm 4.3 \%$, pre- and post-treatment, respectively $(p$-value $=0.217)$. A linear regression analysis showed marginal significant correlation between the absolute mean lung resistivity and hematocrit levels, when all 24 measurements were pooled together, with a Pearson's correlation coefficient of $\mathrm{R}=$ 0.4 (p-value $=0.057)$, see Figure 2 . However, when the change in the mean lung resistivity of a patient was plotted vs. the change in hematocrit readout, a significant linear correlation was found as can be seen in Figure $3(\mathrm{R}=0.7$, $\mathrm{p}$-value $=0.02)$.

\section{DISCUSSION}

In this work, we have correlated the bio-impedance measurements of a group of CPE elderly patients with the hematocrit level, pre- and post treatment with diuretics in order to study the feasibility of the system in monitoring CPE management in these patients. The bio-impedance measurements were performed with a novel hybrid bio-impedance measurement system (PulmoTrace by CardioInspect). Proper management of CPE includes routine monitoring the patient's condition and administration of preload and/or afterload reduction drugs, most notably loop diuretic agents (such as furosemide) in order to remove excessive lung fluids via urine passing. Over dosage of diuretics, however, may generate negative effects such as hypovolemia that reduces cardiac output, as well as hypokalemia due to reduced concentration of potassium in the blood circulation. As a consequence, high efficacy of diuretics treatment for managing CPE is tightly linked to a sensitive and continuous monitoring of the level of lung fluids [11].

At present, pulmonary edema severity level is monitored either invasively or non-invasively. Invasive methods, e.g. single or double thermal die dilution and direct measurement of the capillary wedge pressure, require catheterization, and are therefore intrusive, may result in medical complications and can only be employed in clinical conditions during hospitalization. The thermal die dilution technique is also subject to errors in conditions such as pulmonary vascular perfusion or unilateral lung disease, and despite being considered gold-standard has low accuracy of 20-30\% [12]. Non-invasive methods are comprised of imaging systems, most notably X-ray radiographs, computerized tomography (CT) and magnetic resonance imaging (MRI). While X-ray radiographs are widely practiced, their sensitivity and accuracy are inconsistent and subjective [13,14], especially in cases of coexisting pulmonary diseases [15]. On the other hand, high resolution imaging achieved by CT or MRI provides high accuracy in measuring lung fluids of 


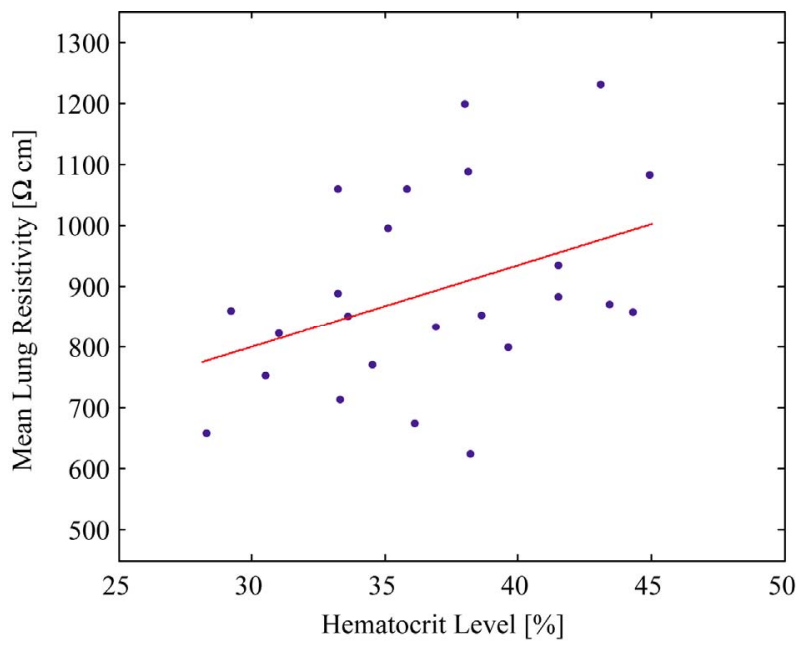

Figure 2. Correlation between mean lung resistivity value and hematocrit $(\mathrm{n}=24, \mathrm{R}=0.4$, $\mathrm{p}$-value $=0.057)$.

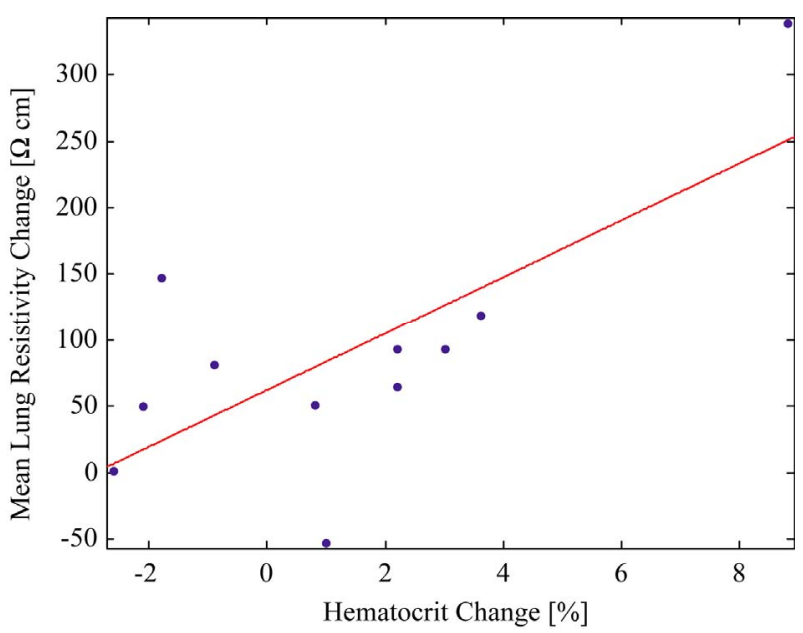

Figure 3. Correlation between mean lung resistivity value change and hematocrit change $(n=12, R=0.7$, $p$-value $=0.02)$.

$\sim 3 \%$, but cannot be utilized routinely due to either high ionizing radiation or the large expenses involved.

The bio-impedance technique has been proposed already in the late 1960's for evaluating the lungs fluid volume. Global measurements of the transthoracic impedance as an indirect marker for lungs fluid level was performed by Pomeranz et al. $(1969,1970)$ on a canine model $[16,17]$. In a common transthoracic measurement configuration, low magnitude and frequency electrical current is injected via a pair of electrodes and the developing voltage between the thorax extremities is simultaneously measured, either by the same pair of electrodes or by a different pair of electrodes ( 2 or 4 electrode configuration, respectively). During the last decades, numerous experimental and clinical studies have employed and improved the global transthoracic impedance measurement for monitoring pulmonary edema, in both ani- mal and human models [18-21]. The technique was also commercialized, e.g. the BioZ system (CardioDynamics, San Diego, CA) that extracts thoracic fluid content and additional hemodynamic and cardiac parameters [22]. Despite the attractiveness of the transthoracic bio-impedance technique for monitoring lungs fluid volume, a major disadvantage is that the technique provides only a global mark of average thoracic impedance, and the relative contributions of the various thoracic organs or even the separate lung lobes to the measured value cannot be assessed. A tomographic variation of the bio-impedance technique for pulmonary function evaluation was first presented in the mid 1980's, with the development of the electrical impedance tomography (EIT) systems [23-25]. These systems can provide a tomographic image of the electrical impedance spatial distribution in a 2D slice of the thorax, or even in a 3D volume. EIT is based on sequential injection of current via different pairs of electrodes that are located along the thoracic perimeter, and measurement of the surface voltages with the rest of the electrodes. The data provided for the various directions of injections enable utilization of reconstruction algorithms (linear or non-linear) that solve the so-called "inverse-problem" of EIT, i.e. the estimation of the internal distribution of impedance that is most probable to result in the set of measured voltages. Various custom-built EIT systems were studied in both animal and human models [26-28]; however, this method seems to suffer from low resolution reconstructions, uneven spatial sensitivity to conductivity perturbations, artifacts arising from numerical reconstruction errors and extremely low signal to noise errors [29]. Most of these limitations are inherent to EIT, due to the mathematical ill-posedeness of the inverse-problem that is solved. The hybrid system that was employed here overcomes the major limitation of EIT systems by employing the inverse problem algorithm to reconstruct only two parameters-the left and the right lung resistivity values. As a result, the reconstruction problem becomes well-posed, and the optimization algorithm is robust, with minimized sensitivity to measurement noises, either geometrical or electrical [6].

In our previous studies we have shown that the system reproducibility on healthy subjects is $<2 \%$, for both within and between tests, with measured signal-to-noise ratio of $\sim 75 \mathrm{~dB}[6]$. We also demonstrated that the system doesn't show dependency on various anthropometric parameters (e.g. body-mass index, age, height and weight). In other studies we found good diagnostic separation capability between healthy subjects and CHF patients, and long-term monitoring of CPE management was validated by tracing the measurements during patient treatment and comparing the bio-impedance measurements to various indirect measures for pulmonary 
congestion e.g. urine output [8] and X-ray diagnosis [9].

In contrast to the hematocrit, the mean lung resistivity value was found to be significantly different between the two measurement phases in the CPE patients (i.e., measurements taken pre- and post-treatment, $\mathrm{p}$-value $=0.016)$. Nevertheless, the results in this study show a significant correlation between the changes in the measured mean lung resistivity value by the hybrid EIT system to changes in hematocrit level following diuretic treatment of CPE patients $(\mathrm{R}=0.7$, $\mathrm{p}$-value $=0.02)$. As a consequence, the validity of lung resistivity measurements by the system is further supported, and it is proposed that bio-impedance measurements such as those taken in this study may be a non-invasive, cost-efficient and patient-friendly supplement or alternative to other monitoring methods for pulmonary congestion.

This study is preliminary and consists of a small number of subjects, resulting in a large variability in the changes of mean lung resistivity. Nevertheless, encouraging results in this study show a significant correlation between the changes in the measured mean lung resistivity value by the hybrid EIT system to changes in hematocrit level following diuretic treatment of CPE patients. A follow-up large scale study that would address these limitations would be a subject of a further research.

\section{ACKNOWLEDGEMENTS}

This work was partially supported by a grant from the ELA KODESZ Institute for Cardiac Physical Sciences and Engineering.

\section{REFERENCES}

[1] Guyton, A.C. (2007) Textbook of medical physiology. W.B. Saunders Company, Philadelphia.

[2] Fromm, R.E. Jr, Varon, J. and Gibbs, L.R. (1995) Congestive heart failure and pulmonary edema for the emergency physician. Journal of Emergency Medicine, 13, 71-87. doi:10.1016/0736-4679(94)00125-1

[3] Figueras, J. and Weil, M.H. (1977) Increases in plasma oncotic pressure during acute Cardiogenic pulmonary edema. Circulation, 55, 195-199.

[4] Figueras, J. and Weil, M.H. (1978) Blood volume prior to and following treatment of acute Cardiogenic pulmonary edema. Circulation, 57, 349-355.

[5] Jahnsen, T., Skovborg, F., Hansen, F., Larsen, J., Nordin, H. and Strom, T. (1983) Variations in blood viscosity in patients with acute Cardiogenic pulmonary oedema treated with frusemide. Scandinavian Journal of Clinical and Laboratory Investigation, 43, 297-300.

[6] Zlochiver, S., Arad, M., Radai, M.M., Barak-Shinar, D., Krief, H., Engelman, T., Ben Yehuda, R., Adunsky, A. and Abboud, S. (2007) A portable bio-impedance system for monitoring lung resistivity. Medical Engineering and Physics, 29, 93-100. doi:10.1016/j.medengphy.2006.02.005

[7] Zlochiver, S., Radai, M.M., Barak-Shinar, D., Ben Gal, T., Yaari, V., Strasberg, B. and Abboud, S. (2005) Moni- toring lung resistivity changes in congestive heart failure patients using the bioimpedance technique. Congestive Heart Failure, 11, 289-293.

doi:10.1111/j.1527-5299.2005.04459.x

[8] Freimark, D., Arad, M., Sokolover, R., Zlochiver, S. and Abboud, S. (2007) Monitoring lung fluid content in CHF patients under intra-venous diuretics treatment using bio-impedance measurements. Physiological Measurement, 28, s269-s277. doi:10.1088/0967-3334/28/7/S20

[9] Arad, M., Zlochiver, S., Davidson, T., Shovman, O., Shoenfeld, Y., Adunsky, A. and Abboud, S. (2009) Estimating pulmonary congestion in elderly patients using bio-impedance technique: correlation with clinical examination and X-ray results. Medical Engineering and Physics, 31, 959-963. doi:10.1016/j.medengphy.2009.05.008

[10] Arad, M., Zlochiver, S., Davidson, T., Shoenfeld, Y., Adunsky, A. and Abboud, S. (2009) The detection of pleural effusion using a parametric EIT technique. Physiological Measurement, 30, 421-428. doi:10.1088/0967-3334/30/4/006

[11] Schuller, D., Mitchell, J.P., Calandrino, F.S. and Schuster, D.P. (1991) Fluid balance during pulmonary edema. Chest, 100, 1068-1075. doi:10.1378/chest.100.4.1068

[12] Brown, B.H., Flewelling, R., Griffiths, H., Harris, N.D., Leathard, A.D., Lu, L., Morice, A.H., Neufeld, G.R., Nopp, P. and Wang, W. (1996) EITS changes following oleic acid induced lung water. Physiological Measurement, 17, A117-A130. doi:10.1088/0967-3334/17/4A/016

[13] Chakko, S., Woska, D., Martinez, H., de Marchena, E., Futterman, L., Kessler, K.M. and Myerberg, R.J. (1991) Clinical, radiographic and hemodynamic correlations in chronic congestive heart failure: conflicting results may lead to inappropriate care. American Journal of Medicine, 90, 353-359.

[14] Liebman, P.R., Philips, E., Weisel, R., Ali, J. and Hechtman, H.B. (1978) Diagnostic value of the portable chest $\mathrm{x}$-ray technic in pulmonary edema. American Journal of Surgery, 135, 604-604. doi:10.1016/0002-9610(78)90045-4

[15] Gehlbach, B.K. and Geppert, E. (2004) The pulmonary manifestations of left heart failure. Chest, 125, 669-682. doi:10.1378/chest.125.2.669

[16] Pomerantz, M., Baumgartner, R., Lauridson, J. and Eiseman, B. (1969) Transthoracic electrical impedance for the early detection of pulmonary edema. Surgery, 66, 260-268.

[17] Pomerantz, M., Delgado, F., and Eiseman, B. (1970) Clinical evaluation of transthoracic electrical impedance as a guide to intrathoracic fluid volumes. Annals of Surgery, 171, 686-694. doi:10.1097/00000658-197005000-00007

[18] Kubicek, W.G., Patterson, R.P. and Witsoe, D.A. (1970) Impedance cardiograph as a non-invasive method of monitoring cardiac function and other parameters of the cardiovascular system. Annals of the New York Academy of Sciences, 170, 724-732. doi:10.1111/j.1749-6632.1970.tb17735.x

[19] Fein, A., Grossman, R.F., Jones, J.G., Goodman, P.C. and Murray, J.F. (1979) Evaluation of transthoracic electrical 
impedance in the diagnosis of pulmonary edema. Circulation, 60, 1156-1160.

[20] Saunders, C.E. (1988) The use of transthoracic electrical bioimpedance in assessing thoracic fluid status in emergency department patients. American Journal of Emergency Medicine, 6, 337-340. doi:10.1016/0735-6757(88)90151-9

[21] Charach, G., Rabinovich, P., Grosskopf, I. and Weintraub, M. (2001) Transthoracic monitoring of the impedance of the right lung in patients with cardiogenic pulmonary edema. Critical Care Medicine, 29, 1137-1144. doi:10.1097/00003246-200106000-00008

[22] Greenberg, B.H., Hermann, D.D., Pranulis, M.F., Lazio, L. and Cloutier, D. (2000) Reproducibility of impedance cardiography hemodynamic measures in clinically stable heart failure patients. Congestive Heart Failure, 6, 74-80. doi:10.1111/j.1527-5299.2000.80140.x

[23] Brown, B.H., Barber, D.C. and Seagar, A.D. (1985) Applied potential tomography: possible clinical applications. Clinical Physics and Physiological Measurement, 6, 109-121. doi:10.1088/0143-0815/6/2/002

[24] Harris, N.D., Suggett, A.J., Barber, D.C. and Brown, B.H. (1987) Applications of applied potential tomography in respiratory medicine. Clinical Physics and Physiological
Measurement, 8, 155-165. doi:10.1088/0143-0815/8/4A/020

[25] Harris, N.D., Suggett, A.J., Barber, D.C. and Brown B.H. (1988) Applied potential tomography: a new technique for monitoring pulmonary function. Clinical Physics and Physiological Measurement, 9, 79-85.

[26] Newell, J.C., Edic, P.M., Ren, X., Larson-Wiseman, J.L. and Danyleiko, M.D. (1996) Assessment of acute pulmonary edema in dogs by electrical impedance imaging. IEEE Transactions on Biomedical Engineering, 43, 133-138. doi:10.1109/10.481982

[27] Frerichs, I., Hahn, G., Schroder, T. and Hellige, G. (1998) Electrical impedance tomography in monitoring experimental lung injury. Intensive Care Medicine, 24, 829836. doi:10.1007/s001340050673

[28] Noble, T.J., Harris, N.D., Morice, A.H., Milnes, P. and Brown, B.H. (2000) Diuretic induced change in lung water assessed by electrical impedance tomography. Physiological Measurement, 21, 155-163. doi:10.1088/0967-3334/21/1/319

[29] Lionheart, W.R. (2004) EIT reconstruction algorithms: pitfalls, challenges and recent developments. Physiological Measurement, 25, 125-142. doi:10.1088/0967-3334/25/1/021 